\title{
Impact of Yukawa-like dimension-five operators on the Georgi-Machacek model
}

\author{
Avik Banerjee, ${ }^{*}$ Gautam Bhattacharyya, ${ }^{\dagger}$ and Nilanjana Kumar ${ }^{\ddagger}$ \\ Saha Institute of Nuclear Physics, HBNI, 1/AF Bidhan Nagar, Kolkata 700064, India
}

(Received 8 January 2019; published 19 February 2019)

\begin{abstract}
We study the effects of including Yukawa-like dimension-five operators in the Georgi-Machacek model where the standard model is augmented with triplet scalars. We focus only on the charged Higgs sector and investigate the constraints arising from radiative $B$-meson decays, neutral $B$-meson mixing and precision measurement of $Z b \bar{b}$ vertex. We observe that the inclusion of the dimension-five operators causes substantial alteration of the limits on the charged Higgs masses and the vacuum expectation value of the triplets, derived otherwise using only the dimension-four operators.
\end{abstract}

DOI: $10.1103 /$ PhysRevD.99.035028

\section{INTRODUCTION}

The standard model (SM) Higgs-like scalar boson, discovered at the Large Hadron Collider (LHC), has not yet provided a full understanding of the dynamics of electroweak symmetry breaking. Many motivated beyond the SM (BSM) scenarios postulate the presence of additional scalars (e.g., SU(2) singlet/doublet/triplet). Exploration of these scalars through direct searches at colliders or through their indirect contributions in various observables is an ongoing exercise. In the absence of any new physics signals at the LHC so far, an effective field theory approach with higher-dimensional operators has been employed in different contexts [1-5]. Construction of effective theories in the singlet and two Higgs doublet extended scenarios has received considerable attention [6-11]. In this paper, we follow a bottom-up approach in studying the triplet-extended Higgs models in the effective field theoretic framework keeping Yukawa-type operators up to dimension five.

For illustration, we choose a particular type of Higgs triplet scenario, known as the Georgi-Machacek (GM) model, which protects custodial symmetry at tree level [12-27]. In addition to the renormalizable Yukawa terms involving the SM doublet Higgs, we include dimension-five operators in the quark sector (especially, the third generation) employing the scalar triplet as well. Note that in two Higgs doublet models the only dimension-five operator is the Weinberg

\footnotetext{
*avik.banerjeesinp@saha.ac.in

'gautam.bhattacharyya@saha.ac.in

*nilanjana.kumar@gmail.com
}

Published by the American Physical Society under the terms of the Creative Commons Attribution 4.0 International license. Further distribution of this work must maintain attribution to the author(s) and the published article's title, journal citation, and DOI. Funded by SCOAP ${ }^{3}$. operator which appears in the leptonic sector generating neutrino Majorana masses [28]. On the other hand, the scalar triplets can couple to the lepton doublets through a renormalizable dimension-four operator leading to type-II seesaw. Further inclusion of dimension-five terms involving the triplets, together with the usual Weinberg operator, would invariably add new twists to neutrino phenomenology. In this paper, however, our main focus is to explore indirect constraints on the charged Higgs sector in the GM model by admitting dimension-five operators that involve the scalar triplets and the heavy third generation quarks.

We note that the presence of higher-dimensional Yukawa-like operators is, in fact, a generic feature of a large class of BSM theories. They can arise, for example, when heavy vector-like fermions are integrated out $[29,30]$. A broad class of composite Higgs models (e.g., little Higgs models, gauge-Higgs unification models, etc.) contains such heavy fermions [31-33]. Instead of appealing to any specific BSM theory, we construct a toy scenario with independent dimension-five operators involving the quarks with unknown $\mathcal{O}(1)$ coefficients, and constrain the parameter space using flavor and electroweak observables, more specifically, $B \rightarrow X_{s} \gamma, B_{s}^{0}-\bar{B}_{s}^{0}$ mixing and $Z b \bar{b}$ vertex. Depending on the relative sign and magnitude of the coefficients of these dimension-five operators, the constraints on the charged Higgs masses can be substantially modified from the existing limits obtained employing the usual dimension-four operators only.

\section{THE SETUP AND THE RELEVANT OPERATORS}

We outline here the salient features of the GM model and construct the dimension-five operators relevant for our discussions. The GM model contains two Higgs triplets with hypercharge $Y=0$ and $Y=1$, in addition to the SM 
Higgs doublet with $Y=1 / 2$. In this scenario, the scalar potential preserves custodial symmetry at tree level without keeping the triplet scalars necessarily inert. This is possible because the Higgs doublet and the triplets can be embedded in the following bi-doublet and bi-triplet representations under $\mathrm{SU}(2)_{\mathrm{L}} \times \mathrm{SU}(2)_{\mathrm{R}}$, respectively, as

$$
\Phi=\left(\begin{array}{cc}
\phi^{0 *} & \phi^{+} \\
-\phi^{-} & \phi^{0}
\end{array}\right), \quad \Delta=\left(\begin{array}{ccc}
\chi^{0 *} & \xi^{+} & \chi^{++} \\
-\chi^{-} & \xi^{0} & \chi^{+} \\
\chi^{--} & -\xi^{-} & \chi^{0}
\end{array}\right)
$$

Details of the scalar potential and its minimization can be found in [17,34]. We assume that $\left\langle\phi^{0}\right\rangle=v_{d} / \sqrt{2}$, $\left\langle\chi^{0}\right\rangle=\left\langle\xi^{0}\right\rangle=v_{t}, \quad$ and $\quad v^{2}=v_{d}^{2}+8 v_{t}^{2} \simeq(246 \mathrm{GeV})^{2}$. Using the standard notation in literature, we define

$$
\tan \beta \equiv \frac{2 \sqrt{2} v_{t}}{v_{d}}
$$

Under the custodial $\mathrm{SU}(2)_{\mathrm{V}}$ one constructs the 5-plet $\left(H_{5}^{ \pm \pm}, H_{5}^{ \pm}, H_{5}^{0}\right)$, triplet $\left(H_{3}^{ \pm}, H_{3}^{0}\right)$ and two singlet $(h, H)$ scalars. With a slight perversion of notation, we shall represent the mass eigenstates after diagonalization using the same symbols. Their expressions in terms of the original fields in Eq. (2.1) are given in [17,34].

We now focus on the couplings of the scalars with the quarks. Since the Yukawa couplings explicitly break custodial symmetry due to different hypercharge assignments for the left- and right-handed fermions, instead of expressing the scalars as bi-doublets and bi-triplets, we rather use $2_{1 / 2}, 3_{0}$, and $3_{1}$ representations under $\mathrm{SU}(2)_{\mathrm{L}} \times \mathrm{U}(1)_{\mathrm{Y}}$, as follows:

$$
\begin{aligned}
& \phi=\left(\begin{array}{c}
\phi^{+} \\
\phi^{0}
\end{array}\right), \quad \xi=\left(\begin{array}{cc}
\xi^{0} / \sqrt{2} & -\xi^{+} \\
-\xi^{-} & -\xi^{0} / \sqrt{2}
\end{array}\right), \\
& \chi=\left(\begin{array}{cc}
\chi^{+} / \sqrt{2} & -\chi^{++} \\
\chi^{0} & -\chi^{+} / \sqrt{2}
\end{array}\right) .
\end{aligned}
$$

The usual dimension-four Yukawa Lagrangian is given by

$$
-\mathcal{L}_{\mathrm{Yuk}}^{(4)}=y_{i j}^{u} \bar{Q}_{L i} \phi^{c} u_{R j}+y_{i j}^{d} \bar{Q}_{L i} \phi d_{R j}+\text { H.c., }
$$

which is not expected to contain the triplets $\xi$ and $\chi$ for group theoretic reason. The triplets, however, can couple to the quarks through dimension-five operators, as

$$
\begin{aligned}
-\mathcal{L}_{\mathrm{Yuk}}^{(5)}= & \frac{c_{5}^{u}}{\Lambda} y_{i j}^{u} \bar{Q}_{L i} \chi^{\dagger} \phi u_{R j}+\frac{c_{5}^{d}}{\Lambda} y_{i j}^{d} \bar{Q}_{L i} \chi \phi^{c} d_{R j} \\
& +\frac{d_{5}^{u}}{\Lambda} y_{i j}^{u} \bar{Q}_{L i} \xi \phi^{c} u_{R j}+\frac{d_{5}^{d}}{\Lambda} y_{i j}^{d} \bar{Q}_{L i} \xi \phi d_{R j}+\text { H.c. },
\end{aligned}
$$

where $\Lambda$ is the cutoff scale of the effective operators. Following the minimal flavor violation hypothesis, the coefficients of dimension-five operators are assumed to be aligned with the Yukawa couplings to avoid stringent constraints from flavor changing neutral current processes. The coefficients $c_{5}^{u, d}$ and $d_{5}^{u, d}$ are taken as $\mathcal{O}(1)$ real numbers, whose exact values and signs depend on the specific underlying UV models. Note that the real coefficients keep the $125 \mathrm{GeV}$ Higgs boson as purely $C P$-even. In this paper, we will treat these coefficients as free parameters. The couplings of the quarks with the physical scalars are displayed in Table I. While the couplings of the 5-plet scalars arise only at the dimension-five level, those involving the triplet and the singlet scalars originate from both dimension-four and dimension-five terms.

TABLE I. The couplings of the quarks with the physical scalars are listed. The relative \pm sign appearing in Feynman rules refer to up/down quarks. Also $s_{\alpha}\left(c_{\alpha}\right) \equiv \sin \alpha(\cos \alpha)$, where $\alpha$ is the mixing angle between the neutral scalars and $t_{\beta}\left(c_{\beta}\right) \equiv \tan \beta(\cos \beta)$. For our calculations, $H_{3,5}^{ \pm}$couplings are relevant.

\begin{tabular}{lc}
\hline \hline Vertices & Feynman rules \\
\hline$h \bar{f} f$ & $-i \frac{m_{f}}{v}\left[\frac{c_{\alpha}}{c_{\beta}}+s_{\alpha}\left(\frac{c_{5}^{f}}{\sqrt{3}} \pm \frac{d_{5}^{f}}{\sqrt{6}}\right) \frac{v}{\Lambda}\right]$ \\
$H \bar{f} f$ & $-i \frac{m_{f}}{v}\left[-\frac{s_{\alpha}}{c_{\beta}}+c_{\alpha}\left(\frac{c_{5}^{f}}{\sqrt{3}} \pm \frac{d_{5}^{f}}{\sqrt{6}}\right) \frac{v}{\Lambda}\right]$ \\
$H_{3}^{0} \bar{f} f$ & $\pm \gamma_{5} \frac{m_{f}}{v}\left[t_{\beta}-\frac{c_{5}^{f}}{\sqrt{2}} \frac{1}{c_{\beta}} \frac{v}{\Lambda}\right]$ \\
$H_{5}^{0} \bar{f} f$ & $-i \frac{m_{f}}{v}\left[\left(\frac{c_{5}^{f}}{\sqrt{6}} \mp \frac{d_{5}^{f}}{\sqrt{3}}\right) \frac{v}{\Lambda}\right]$ \\
$H_{3}^{+} \bar{u} d$ & $-i \frac{\sqrt{2}}{v} V_{u d}\left[\left(t_{\beta}-\frac{1}{c_{\beta}}\left(\frac{c_{5}^{u}}{2 \sqrt{2}}+\frac{d_{5}^{u}}{2}\right) \frac{v}{\Lambda}\right) m_{u} P_{L}-\left(t_{\beta}-\frac{1}{c_{\beta}}\left(\frac{c_{5}^{d}}{2 \sqrt{2}}-\frac{d_{5}^{d}}{2}\right) \frac{v}{\Lambda}\right) m_{d} P_{R}\right]$ \\
$H_{5}^{+} \bar{u} d$ & $i \frac{\sqrt{2}}{v} V_{u d}\left[\left(\frac{c_{5}^{u}}{2 \sqrt{2}}-\frac{d_{5}^{u}}{2}\right) \frac{v}{\Lambda} m_{u} P_{L}-\left(\frac{c_{5}^{d}}{2 \sqrt{2}}+\frac{d_{5}^{d}}{2}\right) \frac{v}{\Lambda} m_{d} P_{R}\right]$ \\
\hline \hline
\end{tabular}




\section{FLAVOR AND ELECTROWEAK PHENOMENOLOGY}

The most significant constraints on the charged Higgs masses and couplings in this setup would come from the radiative $B$ decay, neutral $B$-meson mixing and the precision measurement of the $Z b \bar{b}$ vertex, which we discuss below.

\section{A. $B \rightarrow X_{s} \gamma$ decay}

The latest experimental world average for $\operatorname{Br}^{\exp }(B \rightarrow$ $\left.X_{s} \gamma\right)=(3.32 \pm 0.16) \times 10^{-4}$ [35], while $\operatorname{Br}^{\mathrm{SM}}\left(B \rightarrow X_{s} \gamma\right)=$ $(3.36 \pm 0.23) \times 10^{-4}[36]$. The branching ratio receives large contributions from the charged Higgs couplings via the Wilson coefficient $C_{7}^{\text {eff }}$. The structure of the charged Higgs $\left(H_{i}^{ \pm}\right)$couplings with the quarks is given by

$$
\mathcal{L}=\frac{\sqrt{2}}{v} V_{u d} H_{i}^{+} \bar{u}\left[A_{u}^{i} m_{u} P_{L}-A_{d}^{i} m_{d} P_{R}\right] d+\text { H.c. }
$$

The new physics contributions to $C_{7,8}^{\text {eff }}$ (dominated by the top quark mass) at the matching scale $(\sim 160 \mathrm{GeV}$ [36]) is given by

$$
\delta C_{7,8}^{\mathrm{eff}}=\sum_{i}\left[\frac{\left(A_{t}^{i}\right)^{2}}{3} F_{7,8}^{(1)}\left(x_{i}\right)-A_{t}^{i} A_{b}^{i} F_{7,8}^{(2)}\left(x_{i}\right)\right],
$$

where $x_{i} \equiv m_{t}^{2} / m_{i}^{2}$. Here $i$ takes two values, 3 and 5, which correspond to the scalars $H_{3}^{ \pm}$and $H_{5}^{ \pm}$. We restrict ourselves to leading order in new physics. The functions $F_{7,8}^{(1,2)}\left(x_{i}\right)$ can be found in [37]. The expressions for $A_{t, b}^{i}$ can be read off from Table I. Following [36,38], we have translated the limits on branching ratio to the following range,

$$
-0.063 \leq \delta C_{7}^{\text {eff }}+0.242 \delta C_{8}^{\text {eff }} \leq 0.073,
$$

where we have combined the theoretical and experimental uncertainties in quadrature. With only the dimension-four operators, the new physics part of the GM model always contributes destructively with the SM amplitude leading to a decrease in the overall branching ratio. The dimensionfive operators contribute constructively or destructively depending on the sign of the coefficients. However, since the numerical impact of dimension-five operators is smaller than that of dimension-four operators, the prediction for the overall branching ratio stays reduced compared to the SM expectation.

\section{B. Neutral $B$-meson mixing}

For the sake of demonstration, we show the charged Higgs contributions to the $B_{s}^{0}-\bar{B}_{s}^{0}$ mixing, as it provides slightly stronger constraints than $B_{d}^{0}-\bar{B}_{d}^{0}$ mixing. The primary reason for this is smaller uncertainties in $B_{s}$-system for both experimental measurements and the SM predictions
[35]. The measured value of the mass splitting and its SM prediction in $B_{s}$-system are given by [35]

$$
\begin{aligned}
& \Delta m_{B_{s}}^{\text {exp }}=(17.757 \pm 0.021) \mathrm{ps}^{-1}, \\
& \Delta m_{B_{s}}^{\mathrm{SM}}=(18.3 \pm 2.7) \mathrm{ps}^{-1}
\end{aligned}
$$

We obtain the total contributions to the mass splitting from the $W^{ \pm}$bosons, the Goldstones and the charged Higgs bosons $\left(H_{3}^{ \pm}, H_{5}^{ \pm}\right)$, through the box graphs using standard notations as

$\Delta m_{B_{s}}=\frac{G_{F}^{2} m_{t}^{2}}{24 \pi^{2}}\left(V_{t s}^{*} V_{t b}\right)^{2} f_{B_{s}}^{2} B_{B_{s}} m_{B_{s}} \eta_{B} I_{\mathrm{tot}}\left(x_{W}, x_{i}, x_{j}\right)$,

where $x_{W}=m_{t}^{2} / M_{W}^{2}$, and

$$
\begin{aligned}
I_{\mathrm{tot}}= & I_{W W}\left(x_{W}\right)+\sum_{i, j}\left(A_{t}^{i}\right)^{2}\left(A_{t}^{j}\right)^{2} I_{H_{i} H_{j}}\left(x_{i}, x_{j}\right) \\
& +2 \sum_{i}\left(A_{t}^{i}\right)^{2} I_{W H_{i}}\left(x_{W}, x_{i}\right) .
\end{aligned}
$$

The explicit expressions for the Inami-Lim functions $I_{W W}$, $I_{W H_{i}}$ and $I_{H_{i} H_{i}}$ can be found in $[39,40]$, while we have calculated $I_{H_{i} H_{j}}$ (with $i \neq j$ ), given by

$$
\begin{aligned}
I_{H_{i} H_{j}}= & x_{i} x_{j}\left[\frac{1}{\left(1-x_{i}\right)\left(1-x_{j}\right)}+\frac{\log x_{i}}{\left(x_{i}-x_{j}\right)\left(1-x_{i}\right)^{2}}\right. \\
& \left.+\frac{\log x_{j}}{\left(x_{j}-x_{i}\right)\left(1-x_{j}\right)^{2}}\right] .
\end{aligned}
$$

Normalizing $\Delta m_{B_{s}}$ with respect to its SM prediction, we obtain the following range at $2 \sigma$,

$$
0.675 \leq \frac{\Delta m_{B_{s}}}{\Delta m_{B_{s}}^{\mathrm{SM}}}=\frac{I_{\mathrm{tot}}\left(x_{W}, x_{i}, x_{j}\right)}{I_{W W}\left(x_{W}\right)} \leq 1.265 .
$$

Unlike in the $B \rightarrow X_{s} \gamma$ case, the dimension-four new physics contributions add up constructively with the SM part in neutral meson mixing. However, the dimension-five contributions depend on the sign of $c_{5}^{t}$ and $d_{5}^{t}$.

\section{C. $Z b \bar{b}$ vertex}

One of the most precisely measured electroweak observables is the $Z \rightarrow b \bar{b}$ branching ratio

$$
R_{b}=\frac{\Gamma(Z \rightarrow b \bar{b})}{\Gamma(Z \rightarrow \text { hadrons })},
$$

where $R_{b}^{\exp }=0.21629 \pm 0.00066 \quad[41] \quad$ and $\quad R_{b}^{\mathrm{SM}}=$ $0.21581 \pm 0.00011$ [42]. The modifications in $R_{b}$ due to the charged Higgs contributions at one loop are given by 

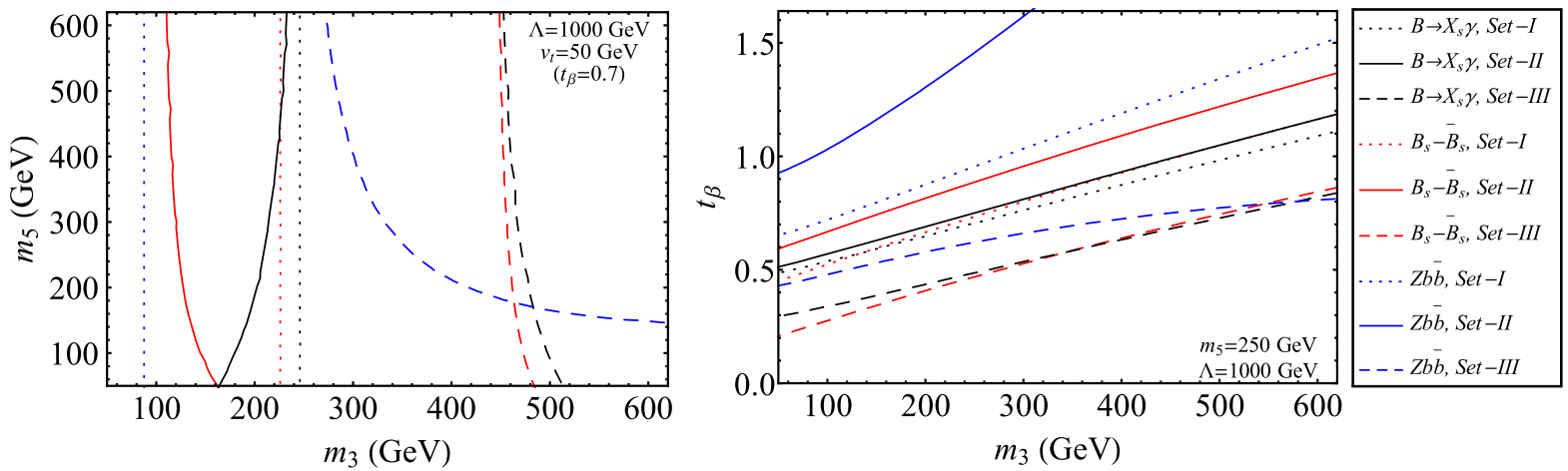

FIG. 1. Exclusion limits on the charged Higgs masses and the triplet vev from $B \rightarrow X_{s} \gamma, B_{s}^{0}-\bar{B}_{s}^{0}$ mixing and $Z b \bar{b}$ vertex for the different sets of benchmark parameters (shown in Table II). In the left panel, regions on the left of each curve are disfavored at $2 \sigma$, while in the right panel the regions above each curve are disfavored at $2 \sigma$. We have fixed $\Lambda=1 \mathrm{TeV}$, and have taken $v_{t}=50 \mathrm{GeV}$ (left panel) and $m_{5}=250 \mathrm{GeV}$ (right panel).

$$
\delta R_{b} \simeq-0.7785 \delta g_{\text {new }}^{L}
$$

Here $\delta g_{\text {new }}^{L}$ is the modification in the $Z b_{L} \bar{b}_{L}$ coupling, calculated from a combination of triangle graphs where $H_{3,5}^{ \pm}$and the charged Goldstones float inside the loop. Their explicit expressions can be found in $[43,44]$. A new type of triangle graph, induced by the dimension-five operators, nevertheless arises in our context. This involves the set $\left\{H_{5}^{ \pm}, W^{\mp}, t\right\}$ inside the loop. Its contribution is given by

$$
\begin{aligned}
\delta g_{\text {new }}^{L}\left(H_{5}^{ \pm}, W^{\mp}, t\right)= & -\frac{g^{2}}{16 \pi^{2}}\left|V_{t b}\right|^{2}\left(\frac{c_{5}^{t}}{2 \sqrt{2}}-\frac{d_{5}^{t}}{2}\right) \\
& \times \frac{v}{\Lambda} s_{\beta} m_{t}^{2} C_{0}\left(m_{t}, M_{W}, m_{5}\right),
\end{aligned}
$$

where $C_{0}\left(m_{t}, M_{W}, m_{5}\right)$ is the usual Passarino-Veltman function [45] and $g$ denotes the $\mathrm{SU}(2)_{\mathrm{L}}$ gauge coupling. This new graph provides a numerically significant interference with the other contributions. We constrain the new physics parameter space by squeezing it in the following $2 \sigma$ range

$$
-0.00086 \leq \delta R_{b} \leq 0.00182
$$

\section{Results}

Before discussing our results, it is worthwhile to recall the existing constraints in the GM model that guided us choose our benchmark values. The oblique $\mathrm{S}$ and $\mathrm{T}$ parameters do not sense the dimension-five Yukawa-like operators at one loop. Nevertheless, they constrain the vacuum expectation value (vev) of the triplet scalars from the dimension-four operators in the gauge sector $[18,34,46]$. The $125 \mathrm{GeV}$ Higgs boson production and decay are of course affected by the dimension-five operators. This, however, involves the neutral Higgs mixing angle $(\alpha)$, which can be tuned to restrict the severity of the contribution [22,46-52]. Nonobservation from direct

TABLE II. Modified lower limits on $m_{3}$ and $m_{5}$ in the presence of dimension-five operators from three observables for different sets of input parameters. Clearly, the Set-I input values correspond to the purely dimension-four case.

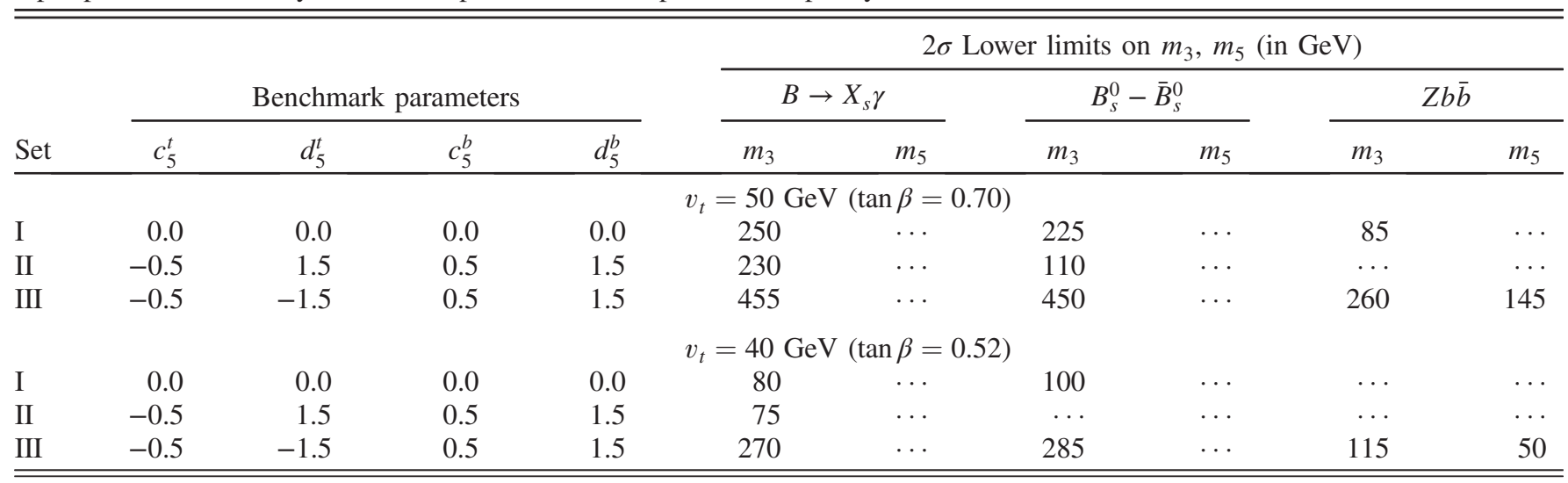




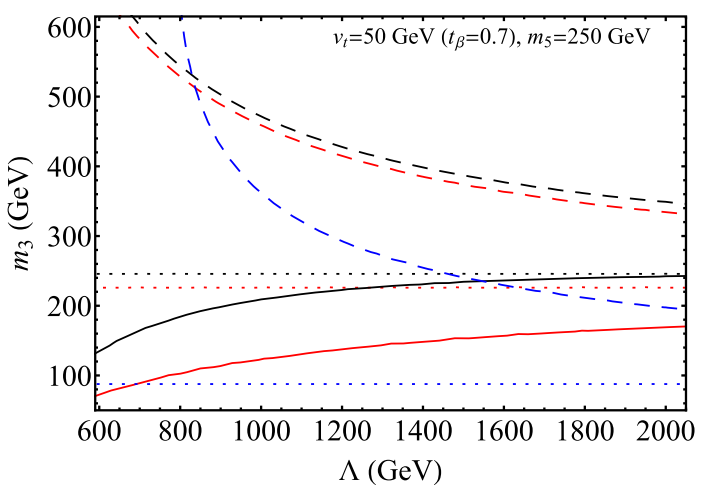

FIG. 2. The cutoff scale $\Lambda$ is varied here fixing $v_{t}=50 \mathrm{GeV}$ and $m_{5}=250 \mathrm{GeV}$. All the regions below each curve are disfavored at $2 \sigma$. The implications of different lines are as in Fig. 1.

searches also restricts the masses of $H^{ \pm}$and $H^{ \pm \pm}$, though the strategies involve several assumptions [50,53-62].

We now discuss our observations. The left panel of Fig. 1 shows the constraints in the plane of the charged Higgs masses coming from the triplet and 5-plet scalars $\left(m_{3}\right.$ and $m_{5}$ ) for a few benchmark points chosen for illustration, while the right panel displays the same in the $\tan \beta-m_{3}$ plane. We draw attention to the substantial contributions from the dimension-five operators when compared to the situation containing only dimension-four terms. The sign and magnitude of the coefficients of the new operators play a crucial role here. In Table II, we present some conservative lower limits on $m_{3}$, and in some cases also on $m_{5}$, for the above mentioned benchmark values of the parameters including the triplet vev. Larger the triplet vev, stronger is the constraint, as expected. Note that the constraints on $m_{5}$ arise only when we consider the dimension-five operators. Among the three sets of observables, only $Z b \bar{b}$ offers reasonable constraints on $m_{5}$. The situation with $B \rightarrow X_{s} \gamma$ has become a little tricky over the last few years $[35,63]$. The experimentally measured central value and the SM prediction have moved in such a way that there exists more space to squeeze our parameters now than a few years ago. Consequently, the limits on the charged Higgs masses in the GM model from $B \rightarrow X_{s} \gamma$ are not as stringent as before [18]. Throughout our analysis we have kept the cutoff scale $\Lambda=1 \mathrm{TeV}$, except in Fig. 2 where we plotted $m_{3}$ against $\Lambda$, fixing other parameters. We emphasize that the constraints we derived on $m_{3}, m_{5}$ and $\tan \beta$, albeit depending on the benchmark values, are both complementary as well as competitive with those obtained from oblique electroweak parameters and direct searches.

\section{CONCLUSIONS AND OUTLOOK}

Two points are worth noting in our analysis. First, we have constructed the dimension-five Yukawa-like effective operators from a bottom-up phenomenological approach in the Georgi-Machacek framework keeping their UV origin unspecified. Second, we demonstrate that for reasonable values of the input parameters these operators significantly modify the limits on the charged Higgs masses. In this context, the dimension-five operators provide a new handle to constrain the mass of the 5-plet charged Higgs $\left(H_{5}^{ \pm}\right)$. All we emphasize is that the limits on the charged Higgs masses derived previously are not infallible once we admit higher-dimensional operators. Therefore, while devising the search strategies, one should not be biased by the previously existing limits.

A natural extension of our study would be to construct similar operators in the leptonic sector, and importantly a new one, given by $l_{L}^{T} C i \tau_{2} \chi \xi l$, together with the standard Weinberg operator $\left(l_{L}^{T} C i \tau_{2} \phi\right)\left(\phi^{T} i \tau_{2} l\right)$. A further extension can be envisaged by constructing the full set of higherdimensional operators in both Yukawa and gauge sectors in an effective theory framework at the expense of introducing more $\mathcal{O}(1)$ parameters which would affect a large pool of observables.

\section{ACKNOWLEDGMENTS}

The authors acknowledge support from the Indo-French Center for Promotion of Advanced Research (IFCPAR/ CEFIPRA, Project No. 5404-2). A. B. acknowledges support from the Department of Atomic Energy, Government of India, and thanks Laboratoire de Physique Théorique, Orsay, and Ecole Polytechnique, Palaiseau, for the hospitality during the initial stages of the work. G. B. acknowledges support of the J.C. Bose National Fellowship from the Department of Science and Technology, Government of India (SERB Grant No. SB/S2/JCB-062/2016). 
[1] W. Buchmuller and D. Wyler, Effective Lagrangian analysis of new interactions and flavor conservation, Nucl. Phys. B268, 621 (1986).

[2] K. Hagiwara, S. Ishihara, R. Szalapski, and D. Zeppenfeld, Low-energy effects of new interactions in the electroweak boson sector, Phys. Rev. D 48, 2182 (1993).

[3] G. F. Giudice, C. Grojean, A. Pomarol, and R. Rattazzi, The strongly-interacting light Higgs, J. High Energy Phys. 06 (2007) 045.

[4] B. Grzadkowski, M. Iskrzynski, M. Misiak, and J. Rosiek, Dimension-six terms in the standard model Lagrangian, J. High Energy Phys. 10 (2010) 085.

[5] J. Ellis, V. Sanz, and T. You, Complete Higgs sector constraints on dimension-6 operators, J. High Energy Phys. 07 (2014) 036.

[6] M. Chala, G. Durieux, C. Grojean, L. de Lima, and O. Matsedonskyi, Minimally extended SILH, J. High Energy Phys. 06 (2017) 088.

[7] J. L. Diaz-Cruz, J. Hernandez-Sanchez, and J. J. Toscano, An effective Lagrangian description of charged Higgs decays $H^{+} \rightarrow W^{+} \gamma, W^{+} Z$ and $W^{+} h_{0}$, Phys. Lett. B 512, 339 (2001).

[8] Y. Kikuta, Y. Okada, and Y. Yamamoto, Structure of dimension-six derivative interactions in pseudo NambuGoldstone N Higgs doublet models, Phys. Rev. D 85, 075021 (2012).

[9] Y. Kikuta and Y. Yamamoto, Derivative interactions and perturbative UV contributions in N Higgs doublet models, Eur. Phys. J. C 76, 297 (2016).

[10] A. Crivellin, M. Ghezzi, and M. Procura, Effective field theory with two Higgs doublets, J. High Energy Phys. 09 (2016) 160.

[11] S. Karmakar and S. Rakshit, Higher dimensional operators in 2HDM, J. High Energy Phys. 10 (2017) 048.

[12] H. Georgi and M. Machacek, Doubly charged HIGGS bosons, Nucl. Phys. B262, 463 (1985).

[13] M. S. Chanowitz and M. Golden, Higgs boson triplets with $M(W)=M(Z) \cos \theta \omega$, Phys. Lett. 165B, 105 (1985).

[14] J. F. Gunion, R. Vega, and J. Wudka, Higgs triplets in the standard model, Phys. Rev. D 42, 1673 (1990).

[15] M. Aoki and S. Kanemura, Unitarity bounds in the Higgs model including triplet fields with custodial symmetry, Phys. Rev. D 77, 095009 (2008).

[16] C.-W. Chiang and K. Yagyu, Testing the custodial symmetry in the Higgs sector of the Georgi-Machacek model, J. High Energy Phys. 01 (2013) 026.

[17] K. Hartling, K. Kumar, and H. E. Logan, The decoupling limit in the Georgi-Machacek model, Phys. Rev. D 90, 015007 (2014).

[18] K. Hartling, K. Kumar, and H. E. Logan, Indirect constraints on the Georgi-Machacek model and implications for Higgs boson couplings, Phys. Rev. D 91, 015013 (2015).

[19] C.-W. Chiang and T. Yamada, Electroweak phase transition in Georgi-Machacek model, Phys. Lett. B 735, 295 (2014).

[20] C.-W. Chiang, S. Kanemura, and K. Yagyu, Novel constraint on the parameter space of the Georgi-Machacek model with current LHC data, Phys. Rev. D 90, 115025 (2014).
[21] H. E. Logan and V. Rentala, All the generalized Georgi-Machacek models, Phys. Rev. D 92, 075011 (2015).

[22] C.-W. Chiang, A.-L. Kuo, and T. Yamada, Searches of exotic Higgs bosons in general mass spectra of the GeorgiMachacek model at the LHC, J. High Energy Phys. 01 (2016) 120.

[23] J. Chang, C.-R. Chen, and C.-W. Chiang, Higgs boson pair productions in the Georgi-Machacek model at the LHC, J. High Energy Phys. 03 (2017) 137.

[24] C. Degrande, K. Hartling, and H. E. Logan, Scalar decays to $\gamma \gamma, Z \gamma$, and $W \gamma$ in the Georgi-Machacek model, Phys. Rev. D 96, 075013 (2017).

[25] S. Blasi, S. De Curtis, and K. Yagyu, Effects of custodial symmetry breaking in the Georgi-Machacek model at high energies, Phys. Rev. D 96, 015001 (2017).

[26] A. Biswas, D. K. Ghosh, A. Shaw, and S. K. Patra, $b \rightarrow c \ell v$ anomalies in light of extended scalar sectors, arXiv: 1801.03375 .

[27] B. Keeshan, H. E. Logan, and T. Pilkington, Custodial symmetry violation in the Georgi-Machacek model, arXiv:1807.11511.

[28] S. Weinberg, Baryon and Lepton Nonconserving Processes, Phys. Rev. Lett. 43, 1566 (1979).

[29] E. Coluccio Leskow, T. A. W. Martin, and A. de la Puente, Vector-like quarks with a scalar triplet, Phys. Lett. B 743, 366 (2015).

[30] M. Chala, M. Ramos, and M. Spannowsky, Gravitational wave and collider probes of extended Higgs sectors with a low cutoff, arXiv:1812.01901.

[31] R. Contino, The Higgs as a composite NambuGoldstone boson, in Physics of the Large and the Small, TASI 09, Proceedings of the Theoretical Advanced Study Institute in Elementary Particle Physics, Boulder, Colorado, USA, 2009 (2011), pp. 235-306, DOI: 10.1142/ 9789814327183_0005.

[32] C. Csaki and P. Tanedo, Beyond the standard model, in Proceedings of the 2013 European School of High-Energy Physics (ESHEP 2013): Paradfurdo, Hungary, 2013 (2015), pp. 169-268, DOI: 10.5170/CERN-2015-004.169.

[33] G. Panico and A. Wulzer, The composite Nambu-Goldstone Higgs, Lect. Notes Phys. 913, 1 (2016).

[34] C.-W. Chiang, A.-L. Kuo, and K. Yagyu, Enhancements of weak gauge boson scattering processes at the CERN LHC, J. High Energy Phys. 10 (2013) 072.

[35] Y. Amhis et al. (HFLAV Collaboration), Averages of $b$ hadron, $c$-hadron, and $\tau$-lepton properties as of summer 2016, Eur. Phys. J. C 77, 895 (2017).

[36] M. Misiak et al., Updated NNLO QCD Predictions for the Weak Radiative B-Meson Decays, Phys. Rev. Lett. 114, 221801 (2015).

[37] M. Ciuchini, G. Degrassi, P. Gambino, and G. F. Giudice, Next-to-leading QCD corrections to $B \rightarrow X_{s} \gamma$ : Standard model and two Higgs doublet model, Nucl. Phys. B527, 21 (1998).

[38] Q.-Y. Hu, X.-Q. Li, and Y.-D. Yang, $B^{0} \rightarrow K^{* 0} \mu^{+} \mu^{-}$decay in the aligned two-Higgs-doublet model, Eur. Phys. J. C 77, 190 (2017).

[39] F. Mahmoudi and O. Stal, Flavor constraints on the twoHiggs-doublet model with general Yukawa couplings, Phys. Rev. D 81, 035016 (2010). 
[40] L. F. Abbott, P. Sikivie, and M. B. Wise, Constraints on charged Higgs couplings, Phys. Rev. D 21, 1393 (1980).

[41] S. Schael et al. (ALEPH, DELPHI, L3, OPAL, and SLD Collaborations, LEP Electroweak Working Group, SLD Electroweak Group, and SLD Heavy Flavour Group), Precision electroweak measurements on the $Z$ resonance, Phys. Rep. 427, 257 (2006).

[42] J. Haller, A. Hoecker, R. Kogler, K. Mönig, T. Peiffer, and J. Stelzer, Update of the global electroweak fit and constraints on two-Higgs-doublet models, Eur. Phys. J. C 78, 675 (2018).

[43] H. E. Haber and H. E. Logan, Radiative corrections to the $Z b \bar{b}$ vertex and constraints on extended Higgs sectors, Phys. Rev. D 62, 015011 (2000).

[44] H. E. Logan, Radiative corrections to the $Z b \bar{b}$ vertex and constraints on extended Higgs sectors, Ph.D. thesis, University of California, Santa Cruz, 1999.

[45] G. Passarino and M. J. G. Veltman, One loop corrections for $e+e-$ annihilation into $\mathrm{mu}+\mathrm{mu}-$ in the Weinberg model, Nucl. Phys. B160, 151 (1979).

[46] S. Kanemura, M. Kikuchi, and K. Yagyu, Probing exotic Higgs sectors from the precise measurement of Higgs boson couplings, Phys. Rev. D 88, 015020 (2013).

[47] H. E. Logan and M.-A. Roy, Higgs couplings in a model with triplets, Phys. Rev. D 82, 115011 (2010).

[48] G. Belanger, B. Dumont, U. Ellwanger, J. F. Gunion, and S. Kraml, Global fit to Higgs signal strengths and couplings and implications for extended Higgs sectors, Phys. Rev. D 88, 075008 (2013).

[49] C.-W. Chiang and K. Tsumura, Properties and searches of the exotic neutral Higgs bosons in the Georgi-Machacek model, J. High Energy Phys. 04 (2015) 113.

[50] B. Li, Z.-L. Han, and Y. Liao, Higgs production at future $e^{+} e^{-}$colliders in the Georgi-Machacek model, J. High Energy Phys. 02 (2018) 007.

[51] C.-W. Chiang, G. Cottin, and O. Eberhardt, Global fits in the Georgi-Machacek model, Phys. Rev. D 99, 015001 (2019).

[52] D. Das and I. Saha, Cornering variants of the GeorgiMachacek model using Higgs precision data, Phys. Rev. D 98, 095010 (2018).

[53] J.-Y. Cen, J.-H. Chen, X.-G. He, G. Li, J.-Y. Su, and W. Wang, Searching for a charged Higgs boson with both
$H^{ \pm} W^{\mp} Z$ and $H^{ \pm} t b$ couplings at the LHC, J. High Energy Phys. 01 (2019) 148.

[54] G. Aad et al. (ATLAS Collaboration), Search for a Charged Higgs Boson Produced in the Vector-Boson Fusion Mode with Decay $H^{ \pm} \rightarrow W^{ \pm} Z$ Using $p p$ Collisions at $\sqrt{s}=$ $8 \mathrm{TeV}$ with the ATLAS Experiment, Phys. Rev. Lett. 114, 231801 (2015).

[55] A. M. Sirunyan et al. (CMS Collaboration), Search for Charged Higgs Bosons Produced via Vector Boson Fusion and Decaying into a Pair of $W$ and $Z$ Bosons Using $p p$ Collisions at $\sqrt{s}=13 \mathrm{TeV}$, Phys. Rev. Lett. 119, 141802 (2017).

[56] C.-W. Chiang, T. Nomura, and K. Tsumura, Search for doubly charged Higgs bosons using the same-sign diboson mode at the LHC, Phys. Rev. D 85, 095023 (2012).

[57] C. Englert, E. Re, and M. Spannowsky, Pinning down Higgs triplets at the LHC, Phys. Rev. D 88, 035024 (2013).

[58] C.-W. Chiang, S. Kanemura, and K. Yagyu, Phenomenology of the Georgi-Machacek model at future electronpositron colliders, Phys. Rev. D 93, 055002 (2016).

[59] H. Sun, X. Luo, W. Wei, and T. Liu, Searching for the doubly-charged Higgs bosons in the Georgi-Machacek model at the electron-proton colliders, Phys. Rev. D 96, 095003 (2017).

[60] CMS Collaboration, A search for doubly-charged Higgs boson production in three and four lepton final states at $\sqrt{s}=13 \mathrm{TeV}, \mathrm{CERN}$, Geneva, Technical Report No. CMS-PAS-HIG-16-036, 2017, https://cds.cern.ch/ record/2242956.

[61] M. Aaboud et al. (ATLAS Collaboration), Search for doubly charged Higgs boson production in multi-lepton final states with the ATLAS detector using proton-proton collisions at $\sqrt{s}=13 \mathrm{TeV}$, Eur. Phys. J. C 78, 199 (2018).

[62] M. Aaboud et al. (ATLAS Collaboration), Search for doubly charged scalar bosons decaying into same-sign $W$ boson pairs with the ATLAS detector, Eur. Phys. J. C 79, 58 (2019).

[63] Y. Amhis et al. (Heavy Flavor Averaging Group (HFAG) Collaboration), Averages of $b$-hadron, $c$-hadron, and $\tau$ lepton properties as of summer 2014, arXiv:1412.7515. 